\title{
Analysis on Internal Motions of the Halo Planetray Nebula, H4-1
}

\author{
M. Otsuka ${ }^{1}$, S. Tamura ${ }^{1}, Y$. Yadoumaru ${ }^{2}, \&$ A.Tajitsu ${ }^{3}$ \\ ${ }^{1}$ Astronomical Institute, Tohoku University, Sendai 980-8578, Japan; \\ ${ }^{2}$ Misato Observatory, Wakayama, 640-1366, Japan; \\ ${ }^{3}$ National Astronomical Observatory, NAOJ, Okayama, 719-0232, Japan
}

Up to now, ten planetary nebulae are regarded as halo planetary nebulae (halo $\mathrm{PNe}$ ). We obtained low- and moderate-dispersion spectra of the spatially resolved halo planetary nebula $\mathrm{H} 4-1$ in the wavelength region of $\lambda \lambda 3700-6800 \AA$ with the Cassegrain Spectrograph of 74-inch telescope at Okayama Astronomical Observatory. In the moderate dispersion observation, we were able to obtain [O III] $\lambda \lambda 4959,5007$ and $\mathrm{H} \alpha$ of $\mathrm{H} 4-1$ at several position angles (P.A.). The image was spatially resolved and its apparent angluar size should be less than $10 . " 0$.

We can analyze the emission line profiles in two dimensions, namely in the direction of dispersion and along the length of slit depending on the position angle. Since the slit width in our observation was $11^{\prime \prime} 8$, two dimensional analysis can give us information on the velocity field around the circumstellar space of the nebula. We show only two samples of the contour plots of the $[\mathrm{O}$ III $] \lambda 5007$ in figure 1 . The contour level are divided into 100 parts between $3 \sigma$ levels of rms and $1 / 10$ of the normalized top of each line profile. We define the expansion velocities of wing components at FWZI (Full Width at Zero Intensity), $V_{F W Z I}$ and estimate it at [O III] $\lambda 5007$ of such wings as $487 \mathrm{kms}^{-1}$ at P.A. $135^{\circ}$ and $610 \mathrm{kms}^{-1}$ at P.A. $180^{\circ}$. Among these values the wing components at P.A. $180^{\circ}$ are clearly blue shifted as a whole. This strongly suggest
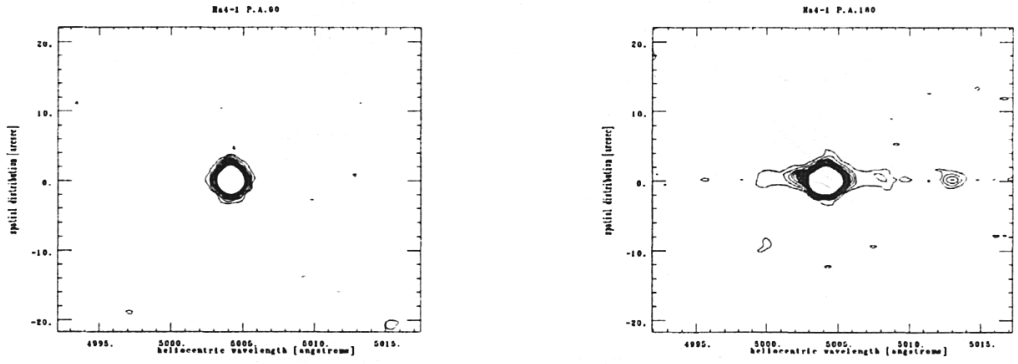

Figure 1. The contour of $[\mathrm{O} \mathrm{III}] \lambda 5007$ at P.A. $90^{\circ}$ (left) and P.A. $180^{\circ}$ (right). The panel at P.A. $180^{\circ}$ clearly shows broad weak components.

the existence of rather high velocity gaseous flow other than the main stream. Morever, this flow can not be explained by the stellar wind which was reported 
by Feibelman (1994, PASP,106,75). As to P.A. $45^{\circ}, 90^{\circ}, 165^{\circ}, 195^{\circ}$, there are no such broadened wing component. Concerning $\mathrm{H} \alpha$, we also detect a broad wing component at P.A. $180^{\circ}$, while there is not at P.A. $90^{\circ}$. The width of the wing component of $\mathrm{H} \alpha$ at P.A. $180^{\circ}$ is estimated as $V_{F W Z I} \sim 442 \mathrm{kms}^{-1}$. The tendency for $V_{F W Z I}$ at P.A. $180^{\circ}$ to be broader than P.A. $90^{\circ}$ is also confirmed in both $\mathrm{H} \alpha$ and [O III] $\lambda 5007$. Therefore, we can confirm an intermediate high velocity flow between the main stream and the stellar wind.

To summarize the measurements on the width of the broadened wing components in connecting with the position angles, we show the resultant values in figure 2(a). We plot them with respect to the centre of system, namely the centre of the main component. In figure 2(b), we also show the relation between the expected FWZIs and the position angles at [O III] $\lambda 5007$. These values can be estimated from observed FWHMs when we assume that they are equivalent to the result of the FWHMs in the single Gaussian fitting. Figure 2(a) reveals a clearly anisotropic feature like a bipolar flow in companion with the figure 2 (b). Given the seeing size (1."5) and the slit width (1." 8$)$ we must keep in the mind that figures 2(a) and 2(b) should be interpreted as the spatial resolution is nearly $2 . \prime 0$. At the time of observation at P.A. $165^{\circ}$ the seeing condition was worse than other positions. Therefore, we suspect an adopted integration time was not enough to reach the level by which we can detect the broad wing.

Further detailed analyses and interpretation are described in a full paper (Otsuka, M., Tamura, S., Yadoumaru, Y., and Tajitsu, A. 2002, to be submitted to PASP.).

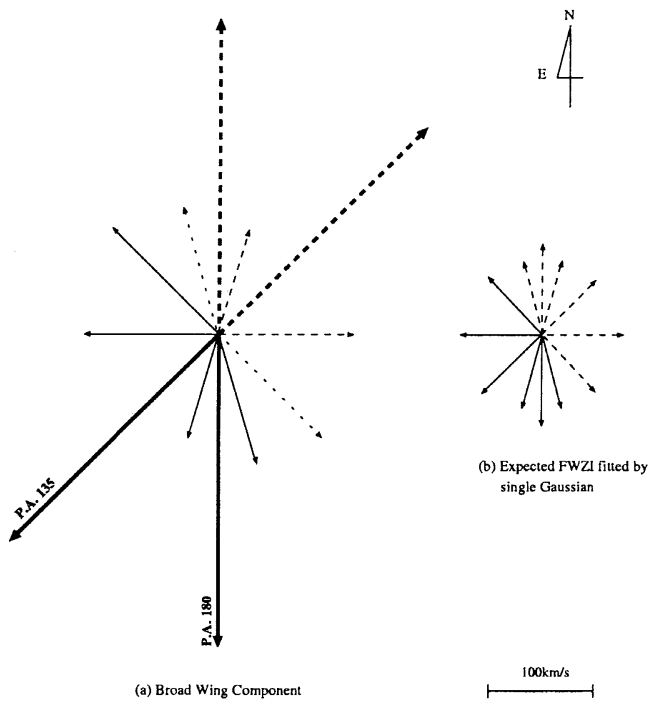

Figure 2. The relation between observed $V_{F W Z I}$ and P.A. at $\left[\mathrm{O}\right.$ III] $\lambda 5007$ ((a), left). The relation between the expected $V_{F W Z I}$ from observed $V_{F W H M}$ and P.A. at [O III] $\lambda 5007$ ((b), right). The solid arrows indicate gas flow toward us, the broken arrows leaving us. Left(a) implies H4-1 has bipolar flow between P.A. $135^{\circ}$ and $180^{\circ}$. On the other hand, right(b) implies the main part of $\mathrm{H} 4-1$ is isotropically expanding. 